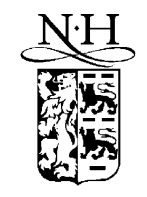

ELSEVIER

\title{
The secondary electron yield of TiZr and TiZrV non-evaporable getter thin film coatings
}

\author{
B. Henrist, N. Hilleret, C. Scheuerlein*, M. Taborelli \\ European Organization for Nuclear Research (CERN), CH-1211, Geneva 23, Switzerland
}

Received 9 August 2000; accepted 21 September 2000

\begin{abstract}
The secondary electron yield (SEY) of two different non-evaporable getter (NEG) samples has been measured 'as received' and after thermal treatment. The investigated NEGs are TiZr and TiZrV thin film coatings of $1 \mu \mathrm{m}$ thickness, which are sputter deposited onto copper substrates. The maximum SEY $\delta_{\max }$ of the air exposed TiZr and TiZrV coating decreases from above 2.0 to below 1.1 during a $2 \mathrm{~h}$ heat treatment at 250 and $200^{\circ} \mathrm{C}$, respectively.

Saturating an activated TiZrV surface under vacuum with the gases typically present in ultra-high vacuum systems increases $\delta_{\max }$ by about 0.1 . Changes in elemental surface composition during the applied heat treatments were monitored by Auger electron spectroscopy (AES). After activation carbon, oxygen and chlorine were detected on the NEG surfaces. The potential of AES for detecting the surface modifications which cause the reduction of SE emission during the applied heat treatments is critically discussed. (C) 2001 Elsevier Science B.V. All rights reserved.
\end{abstract}

PACS: 79.20.H; +82.80.P; +81.65.T

Keywords: Secondary electron yield; Non-evaporable getter; Thin film; Activation; Auger electron spectroscopy; Beam damage

\section{Introduction}

The knowledge of secondary electron emission yields of surfaces subjected to particle bombardment is of great importance for devices whose performance can be hampered by resonant electron multiplication (multipacting). Multipacting is frequently observed in RF cavities for particle acceleration [1] and it can also occur in particle accelerator vacuum systems, in this case driven by the electric field of successive bunches of the particle beam [2].

\footnotetext{
* Corresponding author. Tel.: +41-22-767-8829; fax: +41-22-767-9150.

E-mail address: christian.scheuerlein@cern.ch (C. Scheuerlein).
}

Non-evaporable getter (NEG) thin film coatings are currently being developed at CERN for use in the ultra-high vacuum (UHV) system of the Large Hadron Collider (LHC) [3]. These NEG coatings can be passively activated during a vacuum chamber bakeout. Apart from the required vacuum characteristics, the NEG coatings for use in particle accelerators should also provide a low secondary electron yield (SEY) in order to avoid beam induced multipacting. For the internal surfaces of the LHC vacuum system a critical maximum SEY $\delta_{\max }$ of 1.35 is predicted, above which beam induced multipacting can occur [4].

The investigated NEG coatings consist of $\mathrm{Ti}, \mathrm{Zr}$ and $V$ and the $\delta_{\max }$ values of the atomically clean metals Ti and $\mathrm{Zr}$, found in the literature, are 0.9 and 1.1, 
respectively [5]. However, surfaces which were exposed to air are always covered with contaminating species and as a result the SEY of technical metal surfaces is generally higher than that of the corresponding pure metals.

The SEY of air exposed metals can be decreased by in situ cleaning under vacuum, for instance by a vacuum chamber bake-out. In this article SEY measurements are described which show the SEY variation of air exposed NEG surfaces during in situ thermal treatments.

The applied heat treatments (NEG activation) involve the dissolution of the surface oxides into the bulk of the NEG materials [6]. The variations of the elemental surface composition during this process are monitored by Auger electron spectroscopy (AES).

\section{Experimental}

Throughout this article all electrons emitted upon primary electron (PE) irradiation, regardless of their origin, are called secondary electrons (SE) and the SEY is defined as the number of all emitted SE per incident PE. The maximum SEY value in the SEY versus $\mathrm{PE}$ energy spectrum and the corresponding PE energy are referred to as $\delta_{\max }$ and $E_{\max }$, respectively.

\subsection{Investigated samples}

The investigated samples are TiZr and TiZrV thin film coatings with a thickness of approximately $1 \mu \mathrm{m}$, which are deposited onto chemically polished copper substrates. The coatings are deposited by magnetron sputtering at an argon pressure of about $1 \mathrm{~Pa}$. Scanning electron microscope images of both coatings show that they are smooth on a microscopic scale and no porosity is observed.

The binary TiZr coating is quasi-equiatomic and the ternary TiZrV coating consists of 30 at.\% Ti, 20 at.\% $\mathrm{Zr}$, and 50 at.\% V (determined by energy dispersive $\mathrm{X}$ ray spectroscopy (EDS)). For more information about the NEG thin film coatings see Ref. [3].

After thin film deposition the coating system is vented to ordinary laboratory air and the time during which the samples remain in air before they are introduced into the experimental vacuum systems is less than $24 \mathrm{~h}$. The thin film coatings are not submitted to any chemical cleaning procedures and hence the SEY and AES measurements of the as-received coatings show the influence of the air exposure on atomically clean surfaces.

\subsection{Experimental set-up}

The SEY measurements are carried out in a dedicated experimental set-up, which is described elsewhere [7]. For the calculation of the SEY values the sample-to-ground current and the secondary electron current are measured simultaneously by means of two current amplifiers (Keithley 427), which have an accuracy of $\pm 1 \%$. For all SEY values reported in this paper we estimate an error of \pm 0.03 .

The instrument has been designed to allow SEY measurements using low PE doses because the SEY is very sensitive to electron irradiation [8]. The PE current during the SEY measurements is typically $5 \times 10^{-9} \mathrm{~A}$, incident normal to the sample surface. The pulse length for a single measurement at fixed PE energy is $30 \mathrm{~ms}$ and the beam area is about $1 \mathrm{~mm}^{2}$. The total electron dose for the measurements, which are carried out at 60 different PE energies, is about $10^{-8} \mathrm{C} / \mathrm{mm}^{2}$.

The elemental surface composition as a function of activation temperature is monitored with a special Auger apparatus, which is described in detail in Ref. [9]. The electron spectrometer is a single-pass cylindrical mirror analyser (PHI 15-110B, Physical Electronics) with a coaxial electron gun. The spectra are acquired in the direct $\mathrm{EN}(E)$ mode with a relative energy resolution $\triangle E / E$ of $1.2 \%$ (full width at halfmaximum). Afterwards the spectra are numerically differentiated and the peak-to-peak heights are taken as a measure for peak intensity.

Core electrons are excited by a $2.5 \mathrm{keV}$ electron beam with a current of typically $1 \mu \mathrm{A}$, incident normal to the sample surface. The irradiated sample area is estimated as $0.1 \mathrm{~mm}^{2}$ and the acquisition time for one spectrum is $1 \mathrm{~min}$. Hence, the accumulated PE dose during one Auger spectrum is in the order of $10^{-3} \mathrm{C} /$ $\mathrm{mm}^{2}$, which is five orders of magnitude higher than the PE dose accumulated during the SEY measurements. In order to limit the influence of electron beam damage on the results, each AES measurement is 
carried out on a new sample area, which was not previously exposed to electron irradiation.

In both experiments the samples are tightly clamped to an OFE copper plate by means of four screws in order to keep the sample in good thermal contact with the heatable sample holder. In the SEY system, heating is done by resistive heating of a tungsten-rhenium wire and in the Auger electron spectrometer the sample holder is heated by radiation from a filament. In both cases the sample plate temperature is measured by a thermocouple. The accuracy of the temperature measurements in the AES and SEY experiment are estimated as \pm 3 and $\pm 10^{\circ} \mathrm{C}$, respectively.

The vacuum systems of both instruments are unbaked. The total base pressure before the surface treatments is approximately $10^{-7} \mathrm{~Pa}\left(\mathrm{~N}_{2}\right.$ equivalent $)$, $\mathrm{H}_{2}$ being the dominant gas species followed by water vapour $\left(\mathrm{pH}_{2} \mathrm{O} \sim 2 \times 10^{-8} \mathrm{~Pa}\right)$. During sample heating the total pressure in the experimental vacuum chamber increases up to about $5 \times 10^{-6} \mathrm{~Pa}$, again with $\mathrm{H}_{2}$ as the main gas followed by $\mathrm{H}_{2} \mathrm{O}\left(p \mathrm{H}_{2} \mathrm{O}\right.$ $\left.\sim 5 \times 10^{-7} \mathrm{~Pa}\right)$ and $\mathrm{CH}_{4}\left(p \mathrm{CH}_{4} \sim 5 \times 10^{-7} \mathrm{~Pa}\right)$ The maximum partial pressures of $\mathrm{CO}$ and $\mathrm{CO}_{2}$ during sample heating are in the order of $10^{-7} \mathrm{~Pa}$.

\subsection{Experimental procedures}

All sample surfaces are analysed in the as-received state (after air exposure) and then after in situ heating at $120,160,200,250,300$ and $350^{\circ} \mathrm{C}$. The rate of temperature increase is $10^{\circ} \mathrm{C} / \mathrm{min}$.

In the Auger electron spectrometer the heating time during which each temperature is kept constant is always $1 \mathrm{~h}$, whereas before the SEY measurements the temperature is kept constant during $2 \mathrm{~h}$. SEY measurements of the TiZr coating as a function of the heating time at constant temperature of 160 and $200^{\circ} \mathrm{C}$ show that there is no significant difference in the SEY after 1 and $2 \mathrm{~h}$ heating at these temperatures. The SEY and AES measurements are carried out immediately after the end of the heating cycle when the samples are still hot.

Activated TiZrV samples were exposed to different doses of $\mathrm{H}_{2}, \mathrm{CO}, \mathrm{CO}_{2}$ and $\mathrm{H}_{2} \mathrm{O}$. Before gas injection the sample is always cooled to less than $60^{\circ} \mathrm{C}$. Exposures are carried out in five steps of 3, 30, 300, 3000 and $30000 \mathrm{~L}$ (L: Langmuir, $1 \mathrm{~L}=10^{-6}$ Torr s =
$1.33 \times 10^{-4} \mathrm{Pas}$ ) and the resulting changes of the SEY are recorded.

The exposed gas doses are estimated by measuring the $\mathrm{N}_{2}$ equivalent total pressure in the vacuum system. With a residual gas analyser it can be verified that the injected gas is the dominant species during the exposure. However, at low injection pressures the residual gas species contributes up to $20 \%$ of the total injection pressure.

\section{Results}

\subsection{Influence of the heating temperature on the SEY}

The SEY variation with increasing heating temperature of the TiZrV alloy is shown in Fig. 1. The SEY decreases already strongly after $2 \mathrm{~h}$ heating at $160^{\circ} \mathrm{C}$ and heating the sample to $200^{\circ} \mathrm{C}$ reduces $\delta_{\max }$ further to about 1.1 . The consecutive 250 and $300^{\circ} \mathrm{C}$ heat treatments do not change the SEY significantly.

In order to determine the reproducibility of these measurements the SEY of three TiZrV coatings, which are identical after deposition, have been measured during identical thermal cycles. After $2 \mathrm{~h}$ heating at $200^{\circ} \mathrm{C}, \delta_{\max }$ of the three coatings has been determined as $1.09,1.10$ and 1.18 , and after $2 \mathrm{~h}$ at $250^{\circ} \mathrm{C}$, the corresponding $\delta_{\max }$ values are 1.07, 1.09 and 1.13. In all cases $\delta_{\max }$ is obtained at a PE energy of $350 \mathrm{eV}$.

Fig. 2 shows the influence of the same thermal treatments on the SEY of the TiZr NEG coating. Heating the TiZr NEG coating for $2 \mathrm{~h}$ at $250^{\circ} \mathrm{C}$ decreases its $\delta_{\max }$ to 1.1 . After further $2 \mathrm{~h}$ heating at $300^{\circ} \mathrm{C}, \delta_{\max }$ reaches its lowest value close to unity.

\subsection{Influence of gas exposures on the SEY of the activated TiZrV NEG}

An activated TiZrV sample was exposed to $\mathrm{H}_{2} \mathrm{O}$ and to the gases typically found in accelerator UHV systems, i.e. $\mathrm{H}_{2}, \mathrm{CO}$, and $\mathrm{CO}_{2}$. $\mathrm{CH}_{4}$ is also commonly observed in UHV systems but was not investigated, since it is not pumped by NEGs which operate at room temperature and therefore cannot influence their SEY.

Before the gas exposures the TiZrV thin film was activated during $2 \mathrm{~h}$ at $300^{\circ} \mathrm{C}$ and afterwards it was cooled to $60^{\circ} \mathrm{C}$ within $5 \mathrm{~h}$ in the experimental vacuum system. During this time the NEG surface already 


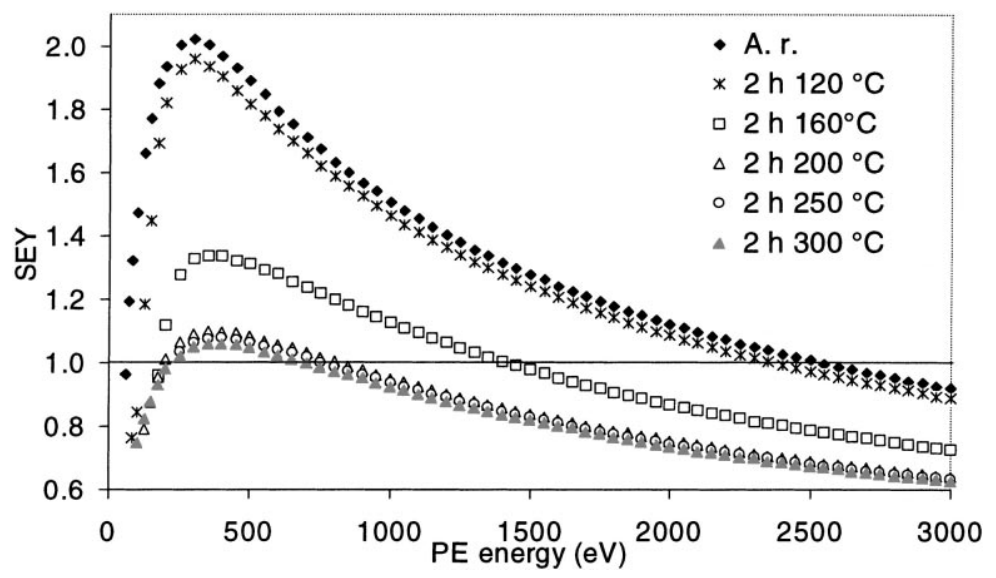

Fig. 1. SEY versus PE energy of the TiZrV NEG coating; as received (A.r.) and after $2 \mathrm{~h}$ heating at 120, 160, 200, 250 and $300^{\circ} \mathrm{C}$.

adsorbs residual gas and as a result sticking probabilities for the injected gases may be influenced by surface contamination.

Fig. 3 shows the influence of $\mathrm{CO}$ exposures on the SEY of the activated TiZrV sample. Changes of the SEY are detected after about $300 \mathrm{~L}$. After $3 \times 10^{3} \mathrm{~L}$, $\delta_{\max }$ is increased by some 0.1 and higher $\mathrm{CO}$ doses increase the SEY only slightly. After $3 \times 10^{4} \mathrm{~L}$ of $\mathrm{CO}$, the SEY does not change further with higher doses.

Gas exposures with $\mathrm{H}_{2}, \mathrm{CO}_{2}$ and $\mathrm{H}_{2} \mathrm{O}$ gave similar results, i.e. changes of the SEY can be detected after about $300 \mathrm{~L}$. After $3000 \mathrm{~L}, \delta_{\max }$ increases by about 0.1 and higher doses have only little effect on the SEY.

\subsection{Influence of the heating temperature on the} elemental surface composition of TiZr and TiZrV

The variation of the elemental surface composition during the thermal treatments is monitored by AES. As an example, the derivative Auger electron spectra of the TiZr thin film coating in the as-received state and after $1 \mathrm{~h}$ heating at different temperatures inside the Auger electron spectrometer are shown in Fig. 4.

The Auger electron spectra show distinct variations when the NEG surface becomes activated. The OKLL peak intensity decreases, the Zr-MNV line shape is initially characteristic of $\mathrm{Zr}$ in $\mathrm{ZrO}_{2}$ and changes to the shape of metallic $\mathrm{Zr}$, the C-KLL peak shape

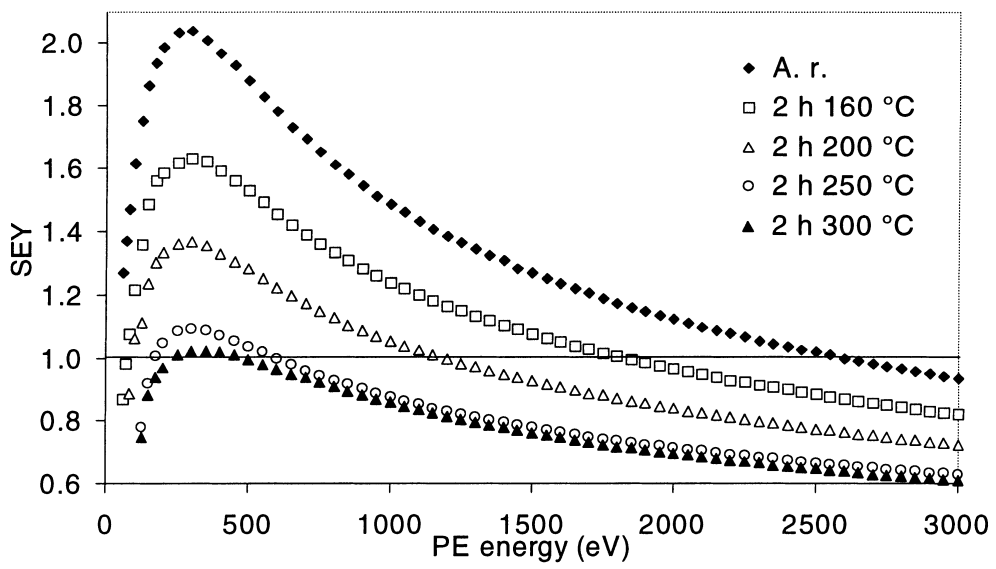

Fig. 2. SEY versus PE energy of the TiZr NEG coating; as received (A.r.) and after $2 \mathrm{~h}$ heating at $160,200,250$ and $300^{\circ} \mathrm{C}$. 


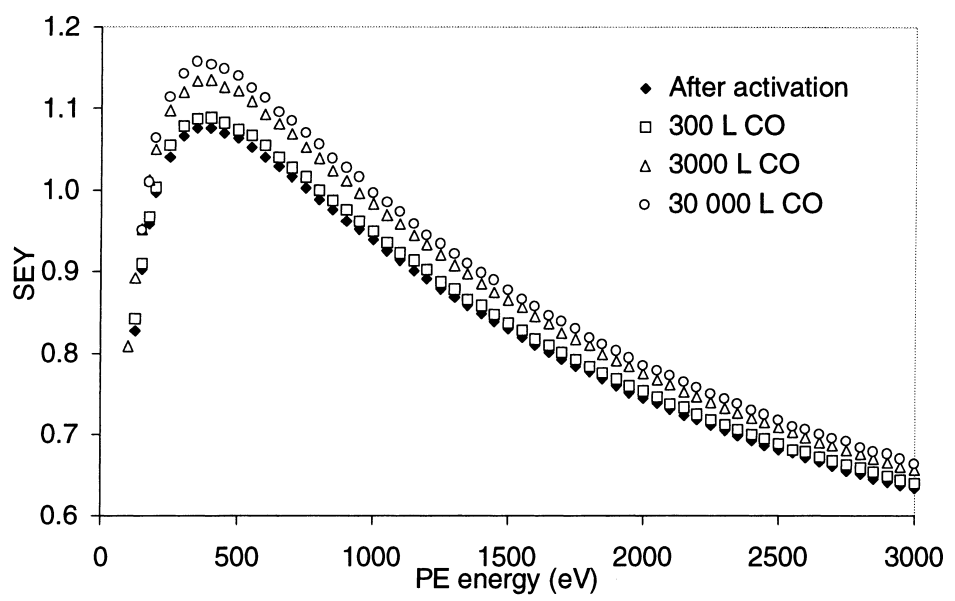

Fig. 3. Influence of CO exposure on the SEY of a TiZrV coating which was activated during $2 \mathrm{~h}$ at $300^{\circ} \mathrm{C}$ and cooled to $60^{\circ} \mathrm{C}$ before the $\mathrm{CO}$ exposure.

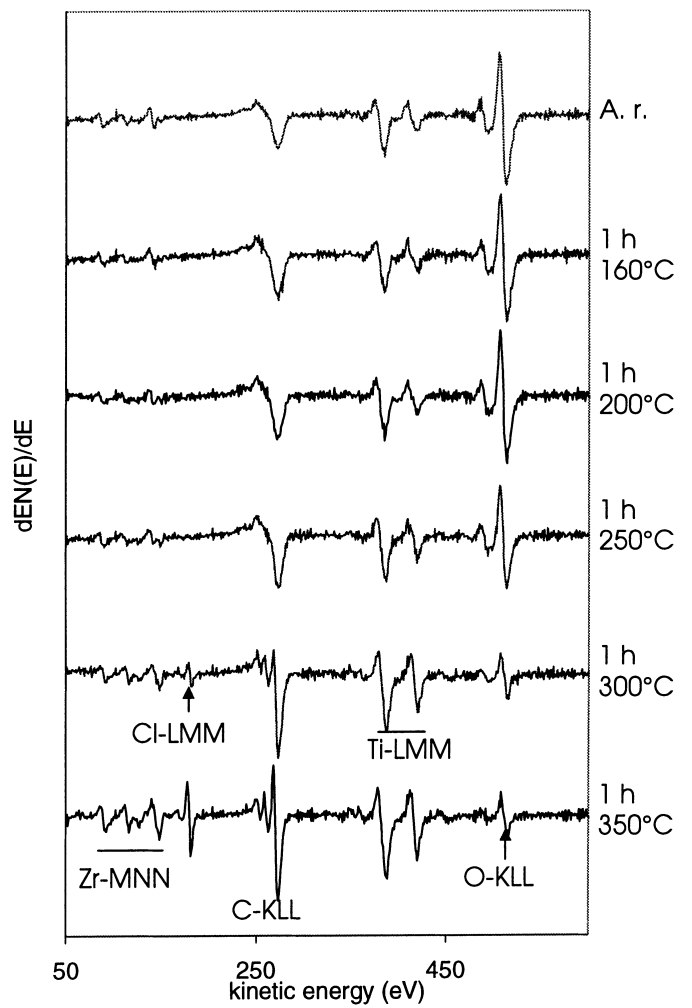

Fig. 4. Derivative $\mathrm{dEN}(E) / \mathrm{d} E$ Auger electron spectra of a TiZr thin film coating as-received and after various thermal treatments. Prior to differentiation the spectra are normalised to the background signal at $600 \mathrm{eV}$ in the direct $\mathrm{EN}(E)$ spectra. The TiZr thin film activation is indicated by a decrease of the O-KLL intensity, by ZrMNV and C-KLL line shape changes and by the appearance of a Cl-LMM peak. changes from graphitic to carbidic and a Cl-LMM peak grows [10]. As an example, the O-KLL intensity variation with increasing heating temperature is shown in Fig. 5.

From the O-KLL intensity decrease and other typical changes in the Auger spectra it can be seen that the oxide layer on the TiZrV surface starts to dissolve into the sample bulk at a temperature between 160 and $200^{\circ} \mathrm{C}$ and on the TiZr surface similar changes occur between 200 and $250^{\circ} \mathrm{C}$.

After activation the O-KLL intensity does not decrease further, possibly because the then chemically reactive NEG surface adsorbs oxygen containing molecules from the residual gas in the Auger electron spectrometer.

\section{Discussion}

\subsection{Influence of thermal treatments on the SEY of the air exposed NEG coatings}

The SEY of the air exposed TiZr and TiZrV NEG thin film coatings can be reduced to values close to those of the pure metals by heating the samples to 250 and $200^{\circ} \mathrm{C}$, respectively. AES measurements reveal that after activation at these temperatures under the experimental conditions the samples are not atomically clean with oxygen and carbon still present on the sample surfaces, possibly due to continuous residual gas adsorption. In addition chlorine is detected on the activated NEG surfaces. 


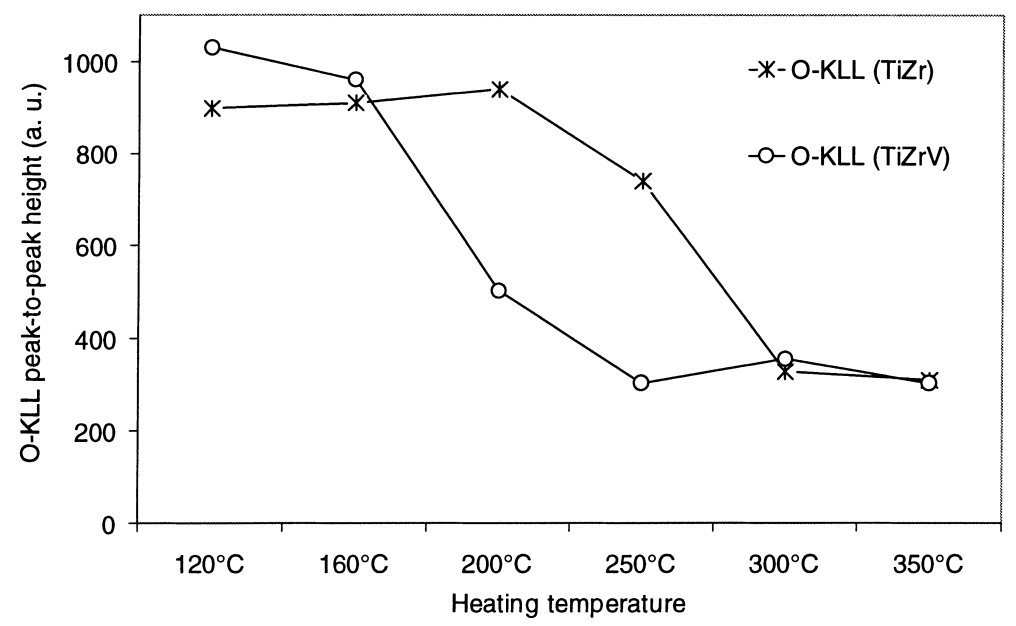

Fig. 5. Influence of $1 \mathrm{~h}$ in situ heating at different temperatures on the oxygen Auger electron intensity (peak-to-peak height) emitted from a TiZr and TiZrV NEG thin film. Prior to differentiation all Auger spectra were normalised to the background signal at $600 \mathrm{eV}$ in the direct $\mathrm{EN}(E)$ spectra.

Strictly the presented results are only valid if exactly the same vacuum conditions as those in the SEY experiment are reproduced during NEG activation. However, since all performed gas exposures increase the SEY of an activated NEG surface one can conclude that if there is a significant influence of surface contamination by adsorption of residual gas in UHV, a higher degree of contamination generally causes a higher SEY. For the prediction of multipacting it is therefore safe to use the presented data, provided that the degree of surface contamination is not higher than during the heating cycles described in this paper.

For a similar decrease of their SEY, typical metals for the construction of particle accelerator vacuum systems (stainless steel, copper, aluminium) require much higher heating temperatures than the investigated NEG coatings. As an example, the SEY of a technical copper surface only decreases from 2.5 to 1.9 during a 24 -h bake-out at $200^{\circ} \mathrm{C}$, which is the maximum temperature to which a copper vacuum chamber can be heated [7].

The measurements described in this article show the SEY variation during the first NEG activation after film deposition and the subsequent air exposure. SEY measurements of a TiZrV thin film coating, which were carried out after several activation/air venting cycles, show that the heating temperature which is required to reduce the SEY to a certain value increases with the number of air exposures of the activated NEG sample.

\subsection{Influence of gas exposures on the SEY of activated NEG samples}

Saturating the activated TiZrV sample with various gases under vacuum conditions increases the SEY but does not have the dramatic effect of an air exposure. As long as the initially activated TiZrV sample is not exposed to air, $\delta_{\max }$ never exceeds 1.3 , regardless of the kind of gas $\left(\mathrm{H}_{2}, \mathrm{CO}, \mathrm{CO}_{2}\right.$, and $\left.\mathrm{H}_{2} \mathrm{O}\right)$ to which the sample is exposed.

After $30000 \mathrm{~L}$ gas exposure one can expect the activated TiZrV surface to be saturated with the injected gases. Only in the case of hydrogen the surface is not saturated because hydrogen diffuses into the NEG bulk even at room temperature.

The different influence of air exposures at atmospheric pressure and controlled gas exposures on the SEY of metals can be explained by the fact that an air exposure of atomically clean metals results in the formation of a surface film which is typically a few nano-meter thick, whereas the uptake of the residual gas species in UHV systems at room temperature virtually stops after the adsorption of the first monolayer. It is has been shown by Bruining [5] and others that secondary electron emission of metals is much less affected by submonolayer adsorption and small 
work function changes than other electron emission processes such as field emission, thermionic emission or photoemission induced by visible light.

\subsection{Usefulness of AES for the detection of the surface properties which determine the SEY}

The AES data presented show that the TiZrV thin film activates at a lower temperature than the TiZr thin film and, accordingly, the SEY of the TiZrV sample can be decreased to a certain value at a lower temperature than that of the TiZr sample.

However, on both samples an important $\delta_{\max }$ decrease from above 2 to below 1.4 already occurs at a temperature at which no significant changes are observed in the corresponding Auger spectra. For the present study, it is therefore of interest to consider whether AES can reveal the surface modifications which cause the SEY decrease of the investigated metal surfaces during the applied heat treatments.

Since AES and SEY measurements are similarly surface sensitive (the average escape depth of SE in metals is approximately $3-5 \mathrm{~nm}$ [11]) one might expect that AES investigations are well suited for this purpose. There are, nevertheless, several important limitations of AES, which are discussed below.

Modifications of the chemical bonds on the surface are in most cases not detectable by the described AES measurements. Hence, the main information gained from the AES data are the Auger peak intensities, which are somehow related to the abundance of the corresponding elements in the probed sample volume. The measured O-KLL peak intensity, as an example, does not represent the oxygen concentration in the oxide but it is an average over the analysed sample depth, which is weighted by a function of the attenuation length. The averaging function changes with the surface composition during the heat treatments, because the attenuation length is material dependent. Taking also into account an estimated accuracy of $\pm 5 \%$ for the presented peak intensity measurements, it is clear that the diffusion of small quantities of oxygen and other species into the metal bulk cannot be detected by AES measurements.

Small changes in the oxygen vacancy concentration in the surface oxide layer may, however, result in significant changes of its electronic structure. The electronic structure has a major influence on the mean escape depth of SE and as a consequence on the SEY.

A further problem of AES in the context with SE emission phenomena is the electron induced surface damage during the AES measurements. Electron induced surface damage is clearly illustrated by its strong effect on the SEY and it is the reason why the SEY measurements are carried out in a dedicated experimental set-up and not in the Auger electron spectrometer. SEY measurements as a function of the incident electron dose show that the SEY of technical metal surfaces is already significantly decreased after an exposure to $10^{-5} \mathrm{C} / \mathrm{mm}^{2}$ of $500 \mathrm{eV}$ electrons. After $10^{-3} \mathrm{C} / \mathrm{mm}^{2}$ the maximum SEY of, for example, a technical copper surface, is drastically changed from an initial value of 2.4 to about 1.1 [12]. The surface properties which determine the SEY are hence drastically changed during Auger measurements since the electron dose accumulated during one of the described AES measurements is about $10^{-3} \mathrm{C} / \mathrm{mm}^{2}$. AES cannot therefore yield information about as-received (air exposed) metal surfaces because it will always analyse a modified surface, which has a much lower SEY than the air exposed metal surface before electron irradiation.

An intrinsic limitation of AES is that this technique cannot detect hydrogen. A variation of the surface hydrogen content may however be important for changes of the SEY, e.g. if the relevant surface variations would involve hydroxides or adsorbed hydrocarbons.

\section{Conclusion}

The SEY of the investigated NEG materials can be strongly decreased by heat treatments at rather low temperatures. The lower the activation temperature of an NEG material, the lower is the heating temperature which is required to decrease the SEY to values close to those of the atomically clean NEG metals.

Saturating an activated NEG under vacuum with the gases typically found in accelerator UHV systems affects the SEY much less than an air exposure.

Despite the similar surface sensitivity of AES and SEY measurements, AES cannot unambiguously identify the surface modifications causing the SEY decrease during the thermal treatment of the NEG 
coatings. This is because of the severe PE induced surface damage, the uncertainties related to the Auger peak intensity measurement and interpretation, a lack of chemical information from AES data and possibly because of the inability of AES to detect hydrogen.

\section{Acknowledgements}

The investigated thin film coatings were produced by P. Costa Pinto, V. Rouzinov and A. Santana (CERN, EST-SM).

\section{References}

[1] H. Lengeler, in: S. Turner (Ed.), CERN Accelerator School Fifth General Accelerator Physics Course, Vol. 2, 1994, p. 791.

[2] O. Gröbner, CERN, LHC Project Report 127, 1997.
[3] C. Benvenuti, J.M. Cazaneuve, P. Chiggiato, F. Cicoira, A. Escudeiro Santana, V. Johanek, V. Ruzinov, J. Fraxedas, Vacuum 53 (1999) 219.

[4] F. Ruggiero, et al., CERN, LHC Project Report 188, 1998.

[5] H. Bruining, Physics and Applications of Secondary Electron Emission, Pergamon Press, London, 1954, p. 39.

[6] C. Benvenuti, P. Chiggiato, F. Cicoira, Y. L'Aminot, J. Vac. Sci. Technol. A 16 (1) (1998) 148.

[7] I. Bojko, N. Hilleret, C. Scheuerlein, J. Vac. Sci. Technol. A 18 (3) (2000) 972.

[8] M. Lavarec, P. Boquet, A. Septier, Comptes Rendus Hebdomadaires des Séances de l'Académie des Sciences, Série B 288 (1978) 77.

[9] C. Benvenuti, R. Cosso, J. Genest, M. Hauer, D. Lacarrere, A. Rijllart, R. Saban, Rev. Sci. Instrum. 69 (1996) 2788.

[10] A.E. Prodromides, C. Scheuerlein, M. Taborelli, Vacuum, in press.

[11] C. Kunz, Synchrotron radiation: overview, in: L. Ley, M. Cardona (Eds.), Photoemission in Solids, Vol. II, Springer, Berlin, 1979, p. 322.

[12] V. Baglin, B. Henrist, N. Hilleret, E. Mercier, C. Scheuerlein, in: Proceedings of the Workshop on LEP-SPS Performance, Chamonix, CERN-SL-2000-007 DI, 2000, p. 130. 